\title{
Self-assembled albumin nanoparticles for combination therapy in prostate cancer
}

\author{
This article was published in the following Dove Press journal: \\ International Journal of Nanomedicine \\ 24 October 2017 \\ Number of times this article has been viewed
}

\author{
Huibo Lian' \\ Jinhui $\mathrm{Wu}^{2}$ \\ Yiqiao $\mathrm{Hu}^{2}$ \\ Hongqian Guo' \\ 'Department of Urology, Drum Tower \\ Hospital, Medical School of Nanjing \\ University, ${ }^{2}$ State Key Laboratory of \\ Pharmaceutical Biotechnology, Medical \\ School of Nanjing University, Nanjing, \\ Jiangsu, People's Republic of China
}

Correspondence: Jinhui Wu State Key Laboratory of Pharmaceutical Biotechnology, Medical School of Nanjing University, 22 Hankou Road, Nanjing 210008 , Jiangsu, People's Republic of China Email wuj@nju.edu.cn

Hongqian Guo

Department of Urology, Drum Tower Hospital, Medical School of Nanjing University, 32I Zhongshan Road, Nanjing 210008 , Jiangsu,

People's Republic of China

Email dr.ghq@nju.edu.cn

\begin{abstract}
Resistance to regular treatment strategies is a big challenge in the treatment of castration-resistant prostate cancer. Combination of photothermal and photodynamic therapy (PTT/PDT) with chemotherapy offers unique advantages over monotherapy alone. However, free drugs, such as photosensitizers and chemotherapeutic agents, lack tumor-targeted accumulation and can be easily eliminated from the body. Moreover, most of the PTT drugs are hydrophobic and their organic solvents have in vivo toxicity, thereby limiting their potential in clinical translation. Herein, simple multifunctional nanoparticles (NPs) using IR780 (a near-infrared dye) and docetaxel (DTX)-loaded nanoplatform based on human serum albumin (HSA) (HSA@ IR780@DTX) was developed for targeted imaging and for PTT/PDT with chemotherapy for the treatment of castration-resistant prostate cancer treatment. In this platform, HSA is a biocompatible nanocarrier that binds to both DTX and IR780. DTX and IR780, as hydrophobic drug, can induce the self-assembly of HSA proteins. Transmission electron microscopic imaging showed that NPs formed by self-assembly are spherical with a smooth surface with a hydrodynamic diameter of 146.5 $\pm 10.8 \mathrm{~nm}$. The cytotoxicity of HSA@IR780@DTX NPs with or without laser irradiation in prostate cancer cells (22RV1) was determined via CCK-8 assay. The antitumor effect of HSA@IR780@DTX plus laser irradiation was better than either HSA@IR780@DTX without laser exposure or single PTT heating induced by HSA@IR780 NPs under near-infrared laser, suggesting a significant combined effect in comparison to monotherapy. Near-infrared fluorescence imaging showed that HSA@IR780@DTX NPs could preferentially accumulate in tumors. In vivo therapeutic efficacy experiment showed that xenografted prostate tumors on mice treated with HSA@IR780@DTX plus near-infrared laser irradiation were completely inhibited, whereas tumors on mice treated with chemotherapy alone (HSA@DTX and HSA@, IR780@DTX without laser) or PTT/PDT alone (HSA@IR780 with laser) showed moderate growth inhibition. Overall, HSA@IR780@DTX NPs showed notable targeting and theranostic potential for the treatment of castration-resistant prostate cancer.
\end{abstract}

Keywords: prostate cancer, combination therapy, albumin nanoparticles, photothermal and photodynamic therapy, chemotherapy

\section{Introduction}

Prostate cancer is the development of cancer in the prostate, a gland in the male reproductive system. It may initially cause no symptoms; however, in later stages it can lead to difficulty urinating, hematuresis, or pain in the bone. Prostate cancer cells may spread to other parts of the body, particularly to the bones and to the lymph nodes. ${ }^{1}$ The treatment strategies available for prostate cancer are multiple. The primary treatments for localized prostate cancer include radical prostatectomy and radiotherapy, whereas for advanced stages, treatments such as chemotherapy, androgen deprivation therapy, and adjuvant radiotherapy are considered. ${ }^{2}$ However, most prostate cancers become 
treatment-resistant after several cycles of therapy, with poor prognosis and low survival. ${ }^{3-6}$ Hence, more effective therapeutic approaches need to be developed in order to improve the treatment and survival of patients with prostate cancer.

Combination therapy is a promising strategy to improve the survival of patients with prostate cancer. Photothermal therapy (PTT) or radiotherapy has been combined with chemotherapy to enhance anticancer efficacy of chemotherapy in a synergistic manner. ${ }^{7-9}$ Among various combination therapies, the combination of PTT with chemotherapy has showed a superior treatment effect in case of prostate cancer. ${ }^{10,11}$ PTT usually utilizes near-infrared (NIR) lightabsorbing photosensitizers to damage tumor cells with laser irradiation by heating. ${ }^{12}$ However, both photosensitizers and chemotherapeutic agents lack tumor-targeted accumulation and can be easily eliminated from the body. Moreover, most PTT agents are hydrophobic, thereby limiting their potential application in clinical practice.

In our previous studies, we developed a simple method to remarkably increase the water solubility of hydrophobic drugs by encapsulating them into human serum albumin (HSA). ${ }^{13,14}$ In this study, a simple method was used to encapsulate hydrophobic IR780 and docetaxel (DTX) into HSA to form HSA nanoparticles (NPs) (HSA@IR780@DTX) for the combination of PTT and PDT with chemotherapy for the enhancement of treatment of castration-resistant prostate cancer (Scheme 1). Albumin, the primary composition of serum proteins, is an excellent nanocarrier for drug delivery because it is biocompatible, biodegradable, has low immunogenicity, and has low toxicity. ${ }^{15}$ DTX, the first-line antitumor chemotherapeutic for prostate cancer, can bind to human serum albumin (HSA) via hydrophobic interactions between the drug molecule and the hydrophobic domain of HSA. ${ }^{16}$ IR780 iodide, an NIR dye, is

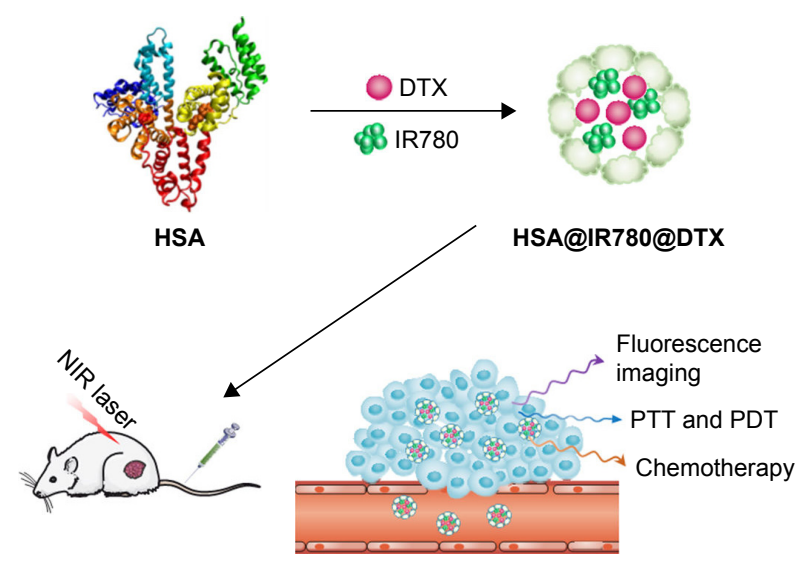

Scheme I A schematic illustration to show the formation of HSA@IR780@DTX nanoparticles by self-assembly between HSA, DTX, and IR780.

Abbreviations: HSA, human serum albumin; DTX, docetaxel; PTT, photothermal therapy; PDT, photodynamic therapy; NIR, near-infrared. a highly hydrophobic agent with specific absorption peak at $780 \mathrm{~nm}$. It has been reported that IR780 could effectively produce heat and generate singlet oxygen after irradiation with $808 \mathrm{~nm}$ laser, suggesting that IR780 can be an ideal agent for PTT and PDT. ${ }^{17,18}$ In our proposed drug delivery system, HSA is a biocompatible and biodegradable carrier platform to deliver DTX, an effective antitumor drug, and IR780, a PTT/PDT agent. Furthermore, IR780 could also serve as a fluorescent imaging probe. DTX is the first-line antitumor chemotherapeutic for castration-resistant prostate cancer. Compared with existing NPs and various formulations used in combination PTTchemotherapy, our self-assembled albumin NPs of DTX are much easier to form and to have potential clinical use for cancer treatment as Abraxane ${ }^{\circledR}$. In this study, we developed simple multifunctional nanoparticles (NPs) using IR780 (a nearinfrared dye)and docetaxel (DTX)-loaded nanoplatform based on human serum albumin (HSA) (HSA@IR780@DTX) for targeted imaging and for PTT/PDT with chemotherapy for the treatment of castration-resistant prostate cancer treatment.

\section{Materials and methods Materials}

HSA solutions were purchased from Baxter. DTX, IR780, and dichlorodihydrofluorescein diacetate $\left(\mathrm{H}_{2} \mathrm{DCFDA}\right)$ were both purchased from Sigma Aldrich. The cell counting kit-8 (CCK-8) was supplied by Dojindo Laboratories (Tokyo, Japan). The obtained HSA@IR780@DTX NPs were characterized by transmission electron microscopy (TEM), dynamic light scattering (DLS), and ultraviolet -visible (UV-Vis) spectrophotometry. It showed simultaneous synergistic PDT/PTT and chemotherapy treatment effect in vitro and in vivo for castration-resistant prostate cancer. Deionized water was used throughout the experiments. We purchased 22RV1 cell lines from the Institute of Biochemistry and Cell Biology, Shanghai Institute of Life Sciences, Chinese Academy of Sciences. All the nude mice were purchased from Yangzhou University Medical Center, and the weight of each mouse was between 20 and $22 \mathrm{~g}$.

\section{Preparation and characterization of HSA@IR780@DTX NPs}

We dissolved $200 \mathrm{mg}$ of HSA in $100 \mathrm{~mL}$ of deionized water with constant stirring. Next, 2-mercaptoethanol was added to expose the hydrophobic region of HSA. Then, IR780 and DTX (2 and $30 \mathrm{mg} / \mathrm{mL}$, respectively) were dissolved in dimethylformamide by slow addition, which denatures the protein and stirred until they were completely dissolved. We performed ultrafiltration of the obtained dispersions to remove 2-mercaptoethanol and unbound HSA or DTX (the molecular 
weight was $60 \mathrm{kDa}$ ) using an ultrafiltration membrane with the molecular weight cut-off of $\sim 100 \mathrm{kDa}$. Then, the solution was filtered through $220 \mathrm{~nm}$ filter membrane. Finally, the NP dispersions (namely,HSA@IR780,HSA@DTX, and HSA@ IR780@DTX NPs) were stored at $4{ }^{\circ} \mathrm{C}$. The amount of IR780 or DTX in NPs was determined by UV-Vis absorption spectra and HPLC. The morphology and particle size of NPs were analyzed by TEM (Hitachi H-7650). The NPs were dried on a copper grid coated with amorphous carbon and then observed at $200 \mathrm{kV}$. The NPs were also coated in a cathodic evaporator with a fine gold player and observed on a scanning electron microscope (Hitachi S-3400N). UV-Vis spectra were measured by a UV-Vis spectrophotometer (UV2450, Shimadzu Corporation). The diameter of NPs was measured by a DLS analyzer (Brookhaven Instruments Corporation, NY, USA). The final concentration of IR780 or DTX in the NP solutions was $1 \mathrm{mg} / \mathrm{mL}$ and $10 \mathrm{mg} / \mathrm{mL}$, respectively. To evaluate the photothermal effects of NPs in aqueous solution, NPs were exposed to $808 \mathrm{~nm}$ wavelength laser irradiation $\left(1 \mathrm{~W} / \mathrm{cm}^{2}\right)$ with the illumination direction from the top to the bottom of the cuvette. The negative control was an equivalent amount of water with the same laser irradiation. The temperature of solution was determined with a thermal probe at different time points.

\section{ROS generation by HSA@IR780 and HSA@IR780@DTX NPs in cancer cells}

$\mathrm{H}_{2}$ DCFDA acts as a fluorescent probe for ROS in cells, which is non-fluorescent before penetrating into cells. Once entering the cells, the diacetate group of $\mathrm{H}_{2}$ DCFDA would be removed by intracellular esterases. The remaining part would be oxidized rapidly and emit green fluorescence in the presence of ROS. Based on this principle, pre-seeded 22RV1 cells in a confocal 12-well plate were co-incubated with HSA@IR780 NPs, HSA@DTXNPs,HSA@IR780@DTX NPs dispersions, or with PBS and $\mathrm{H}_{2}$ DCFDA $(40 \mu \mathrm{M})$ for $4 \mathrm{~h}$ in an atmosphere of $37^{\circ} \mathrm{C}$ and $5 \% \mathrm{CO}_{2}$. Subsequently, the cells were washed with phosphate buffer saline (PBS) and the central region in the 12-well plate was exposed to $808 \mathrm{~nm}$ wavelength laser irradiation $\left(1 \mathrm{~W} / \mathrm{m}^{2}\right)$ for $5 \mathrm{~min}$. Fluorescence images in the irradiation region were immediately captured on a confocal fluorescence microscope (Olympus FV1000) using an excitation of $504 \mathrm{~nm}$ and an emission of 510-560 nm.

\section{Cellular uptake of HSA@IR780 NPs and HSA@IR780@DTXNPs}

We seeded 22RV1 cells onto a glass-bottom dish (glass diameter $10 \mathrm{~mm}$ ) at a density of $1.0 \times 10^{4}$ cells/dish and cultured for $24 \mathrm{~h}$ in an atmosphere of $37^{\circ} \mathrm{C}$ in $5 \%$ humidified
$\mathrm{CO}_{2}$. After $24 \mathrm{~h}$, the medium was changed to the fresh medium containing HSA@IR780 andHSA@IR780@DTX NPs. After $2 \mathrm{~h}$ incubation, the cells were washed thrice with PBS and fixed with $4 \%$ paraformaldehyde solution for $20 \mathrm{~min}$, and then stained with DAPI for $15 \mathrm{~min}$. Finally, the cells were observed by confocal laser scanning microscope (CLSM, Leica TCS SP5, Germany). The excitation wavelength of IR780 was 633 $\mathrm{nm}$ and the emission spectrum recorded was between 700 and $800 \mathrm{~nm}$. In addition, for the cellular uptake experiment by flow cytometry, 22RV1 cells were seeded in 6-well plate at a density of $5 \times 10^{5} /$ well. The treatment was the same as cell uptake mentioned above. Then, the cells were washed with PBS three times, centrifuged, re-suspended in $500 \mu \mathrm{L}$ PBS, and analyzed by flow cytometry. The fluorescence of IR780 was collected on the FL4-H channel.

\section{In vitro therapeutic efficacy of NPs}

The cytotoxicity of HSA@IR780 and HSA@IR780@DTX NPs with or without light irradiation in 22RV1 cells was determined via CCK-8 assay. Briefly, the cells were seeded in a 96-well plate at a density of $5 \times 10^{3}$ cells/well in $100 \mu \mathrm{L}$ DMEM containing $10 \%$ FBS. After incubation for $24 \mathrm{~h}$, samples with various concentrations of NPs were added to each well. After $2 \mathrm{~h}$, the medium was replaced with $100 \mu \mathrm{L}$ fresh medium, and the cells were irradiated with a $1 \mathrm{~W} / \mathrm{cm}^{2}$ 808 -nm laser for $2.5 \mathrm{~min}$. Control cells were treated identically but not irradiated with the laser. Subsequently, the cells were incubated for $24 \mathrm{~h}$, and CCK-8 assay was performed to quantify the cell viability. The cell viability was defined as the percentage of surviving cells versus untreated cells.

\section{In vivo NIR fluorescence imaging of HSA@IR780@DTX NPs}

In vivo imaging and biodistribution analysis

When the size of the tumor in mice reached about 100-200 mm³ , the HSA@IR780@DTX NPs (3 mg IR780/kg body weight) were injected via tail vein $(\mathrm{n}=6)$. Images were taken at $0,12,24$, and $48 \mathrm{~h}$ after injection using the in vivo imaging system (IVIS Lumina XR III, USA). Three of the $\mathrm{BALB} / \mathrm{c}$ mice were sacrificed at $48 \mathrm{~h}$ after injection. Then the organs including heart, liver, spleen, lung, kidney, brain, and tumor tissue were collected for imaging and semi-quantitative biodistribution analysis by the imaging system. The excitation wavelength employed for IR780 was $735 \mathrm{~nm}$ and the emission spectrum was at $780-900 \mathrm{~nm}$.

\section{Therapeutic efficacy of NPs in vivo}

Male nude mice received care in compliance with the guidelines outlined in the Guide for the Care and Use of Laboratory 
Animals and were used in accordance with the regulations of the Institutional Animal Care and Use Committee (IACUC) of Nanjing University. All animal tests and experimental procedures were approved by the Administration Committee of Experimental Animals in Jiangsu Province and the Ethics Committee of Nanjing University.

To establish the tumor model, 22RV1 cells $\left(5 \times 10^{6}\right)$ were administered via subcutaneous injection into the right flank of the mice. Treatments were initiated when the tumor volumes reached 100-200 $\mathrm{mm}^{3}$. To evaluate the thermal effect of NPs in vivo, $100 \mu \mathrm{L}$ of HSA@IR780 and HSA@IR780@ DTX NPs ( $5 \mathrm{mg} / \mathrm{kg}$, concentration of IR780) was injected into the tumor-bearing mice by vein. After $24 \mathrm{~h}$, an infrared (IR) thermal camera (Fluke, USA) was used to monitor the temperature change when the tumors were exposed to $808 \mathrm{~nm}$ laser power density of $1 \mathrm{~W} / \mathrm{cm}^{2}$ at $1 \mathrm{~min}$ intervals for a total of $4 \mathrm{~min}$. When the tumor volumes reached about $100-200 \mathrm{~mm}^{3}$, the mice were divided into seven groups and treated with PBS, HSA@DTX,HSA@IR780,orHSA@IR780@DTX with or without laser irradiation (IR780 dose: $5 \mathrm{mg} / \mathrm{kg}$ ). All samples were given by intravenous injection via tail vein. The day of administration was set as day 0 and $24 \mathrm{~h}$ later, the tumors were exposed to $808 \mathrm{~nm}$ laser $\left(1 \mathrm{~W} / \mathrm{cm}^{2}\right)$ for $4 \mathrm{~min}$. Tumor volumes were calculated using the following formula: tumor volume $=$ length $\times$ width $2 \times 0.5$. Tumor sizes and body weights were measured every 3 days for the duration of the experiment. After more than 2 weeks of treatment, the animals were sacrificed and tumors and primary organs (heart, liver, spleen, lung, and kidney) were harvested for H\&E staining.

\section{Results and discussion}

\section{Preparation and characterization of} HSA@IR780@DTXNPs

HSA@IR780,HSA@DTX NPs, and HSA@IR780@DTX NPs were prepared according to the previous methods. ${ }^{13}$ First,

A

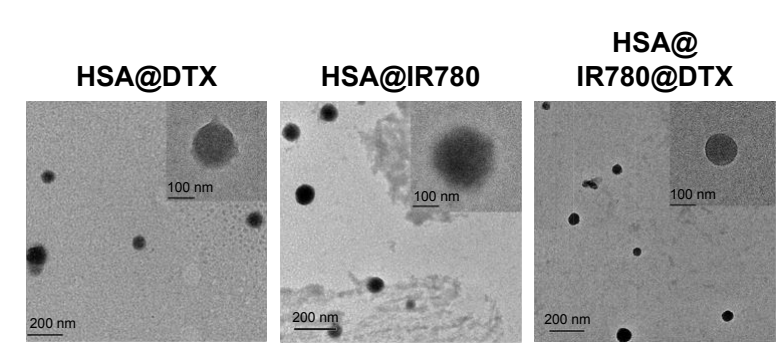

the disulfide bond of has was broken by 2-mercaptoenthanol and the hydrophobic domains of HSA were exposed. IR780 and DTX are strong hydrophobic molecules, tending to integrate with the exposed hydrophobic parts of HSA to form the NPs. While the hydrophilic groups of HSA were exposed to water, the mixture of IR780, DTX, IR780, and DTX were enclosed in the hydrophobic groups of HSA. Then, the HSA@IR780,HSA@DTX NPs, and HSA@IR780@DTX NPs were formed. The optimized formulation prepared at a molar ratio of HSA:IR780 (1:10), HSA:DTX (1:30), and HSA:IR780:DTX (1:10:30) were selected and used in our following experiments. The obtained NP dispersions were ultrafiltered to remove excess molecules. The IR780 and DTX content in the HSA@IR780@DTX formulation were measured to be $2.3 \%$ and $6.5 \%$ (the loading efficiencies of IR780 and DTX were $76.9 \%$ and $39.4 \%$ ), respectively, by high-performance liquid chromatography (HPLC).

We characterized the obtained HSA@IR780,HSA@DTX NPs, and HSA@IR780@DTX NPs. TEM imaging showed that all NPs formed by self-assembly were spherical with a smooth surface (Figure 1A). Hydrodynamic diameters of HSA@IR780,HSA@DTX NPs, and HSA@IR780@DTX were measured by DLS. The mean diameter was found to be $142 \pm 10.5,170.1 \pm 17.9$, and $146.5 \pm 10.8$, respectively (Figure 1B). In the preparation of different kinds of NPs, HSA as a nanocarrier was excess for HSA@DTX NPs and HSA@ IR780@DTX NPs; thus, when more hydrophobic drugs were encapsulated in HSA, it may produce more NPs rather than increase particle size. As shown in Figure 1C, UV-Vis spectra indicated that HSA-DTX solution had no absorption in the NIR region. Compared with free IR780, HSA@ IR780 and HSA@IR780@DTX showed slightly red-shifted absorbance peak probably due to the molecular conformation change after IR780 is complexed with the albumin. Next, we investigated the photothermal profile of the NPs.HSA@

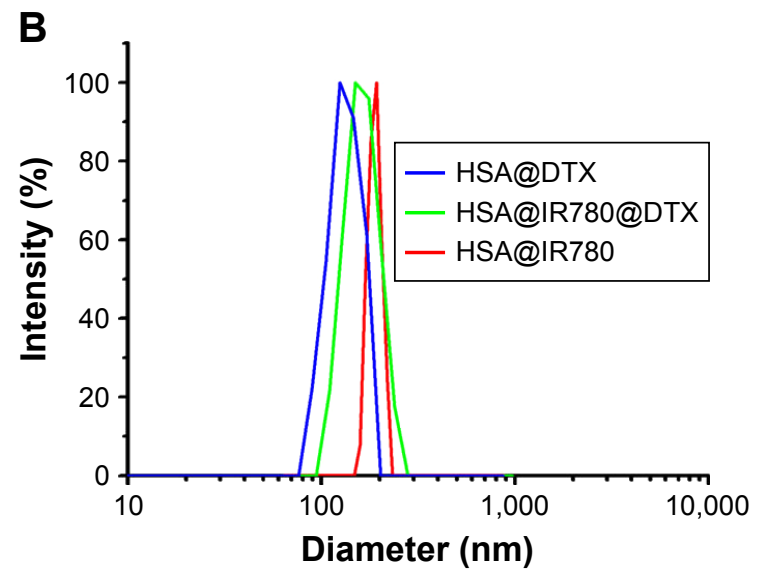

Figure I (Continued) 

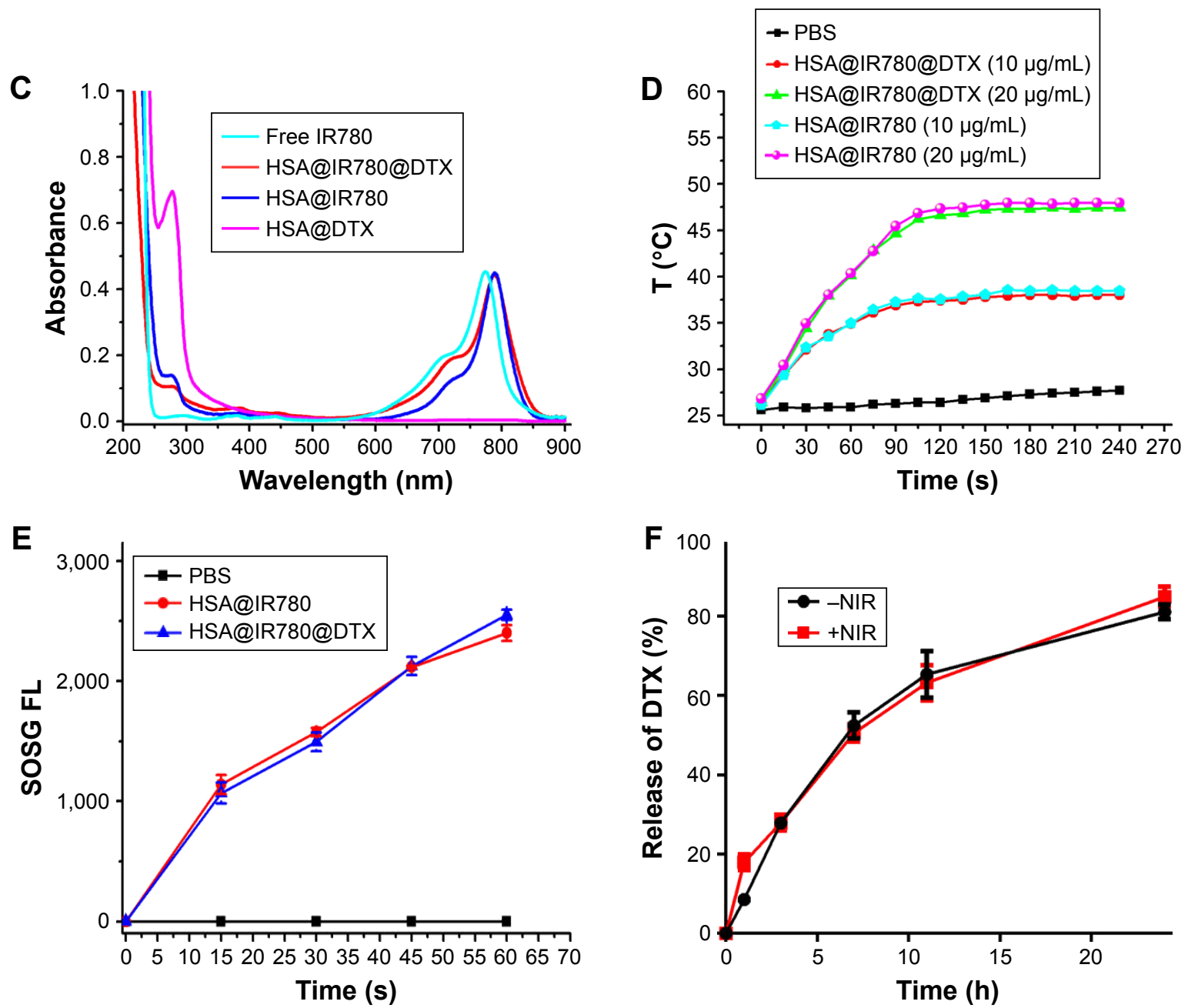

Figure I Preparation and characterization of HSA-ICG-DTX complex. (A) TEM images of HAS@DTX, HSA@IR780, and HSA@IR780@DTX. (B) Hydrodynamic diameters of HAS@DTX, HSA@IR780, and HSA@IR780@DTX measured by DLS. (C) UV-Vis-NIR spectra of Free IR780, HAS@DTX, HSA@IR780, and HSA@IR780@ DTX. (D) Temperature change of PBS, HSA@IR780, and HSA@IR780@DTX in the irradiation of a NIR laser (808 nm) with a power density of I W/cm². (E) Singlet oxygen generation of NPs after NIR laser irradiation. (F) Release profiles of HSA@IR780@DTX with or without laser irradiation.

Abbreviations: DTX, docetaxel; FL, fluorescence; HSA, human serum albumin; NIR, near-infrared; PBS, phosphate buffer saline; SOSG, singlet oxygen sensor green; ICG, indocyanine green.

IR780 and HSA@IR780@DTX solutions showed the similar photothermal profile under the $808 \mathrm{~nm}$ laser irradiation $\left(1 \mathrm{~W} / \mathrm{cm}^{2}\right)$ (Figure 1D). The temperature change of NPs was in a concentration-dependent manner. The temperature of the HSA@IR780@DTX NPs (20 $\mu \mathrm{g} / \mathrm{mL})$ increased from room temperature $\left(26.4^{\circ} \mathrm{C}\right)$ to $45^{\circ} \mathrm{C}$ in $90 \mathrm{~s}$ under laser irradiation. The maximum temperature was found to be $47.5^{\circ} \mathrm{C}$. Such observed temperatures are high enough to cause irreversible hyperthermia damage to tumor cells. To verify the photodynamic effects of NPs, the singlet oxygen generation of NPs is manifested by the fluorescence intensity of singlet oxygen sensor green (SOSG). The fluorescence intensity of NPs gradually increased after being exposed to $808 \mathrm{~nm}$ laser irradiation, indicating the generation of singlet oxygen generated (Figure 1E). The release profiles of DTX from HSA@IR780@ DTX nanocomplexes with or without NIR laser irradiation were also investigated (Figure 1F). As shown in Figure 1, there was no significant difference between the release profile with NIR laser irradiation and without NIR laser irradiation.

\section{ROS generation of HSA@IR780 and HSA@IR780@DTX NPs in cancer cells}

We studied ROS generation under exposure to $808 \mathrm{~nm}$ laser irradiation. ROS generation induced by IR780 can oxidize $\mathrm{H}_{2}$ DCFDA to produce green fluorescence. As shown in Figure 2, fluorescence can be barely observed in cells treated with HSA@IR780 and HSA@IR780@DTX NPs without laser irradiation. However, cells treated with HSA@IR780 and HSA@IR780@DTX NPs plus NIR irradiation exhibited obvious green fluorescence, demonstrating an amount of ROS generation in cells and further predicting the application of NPs for PDT therapy. 

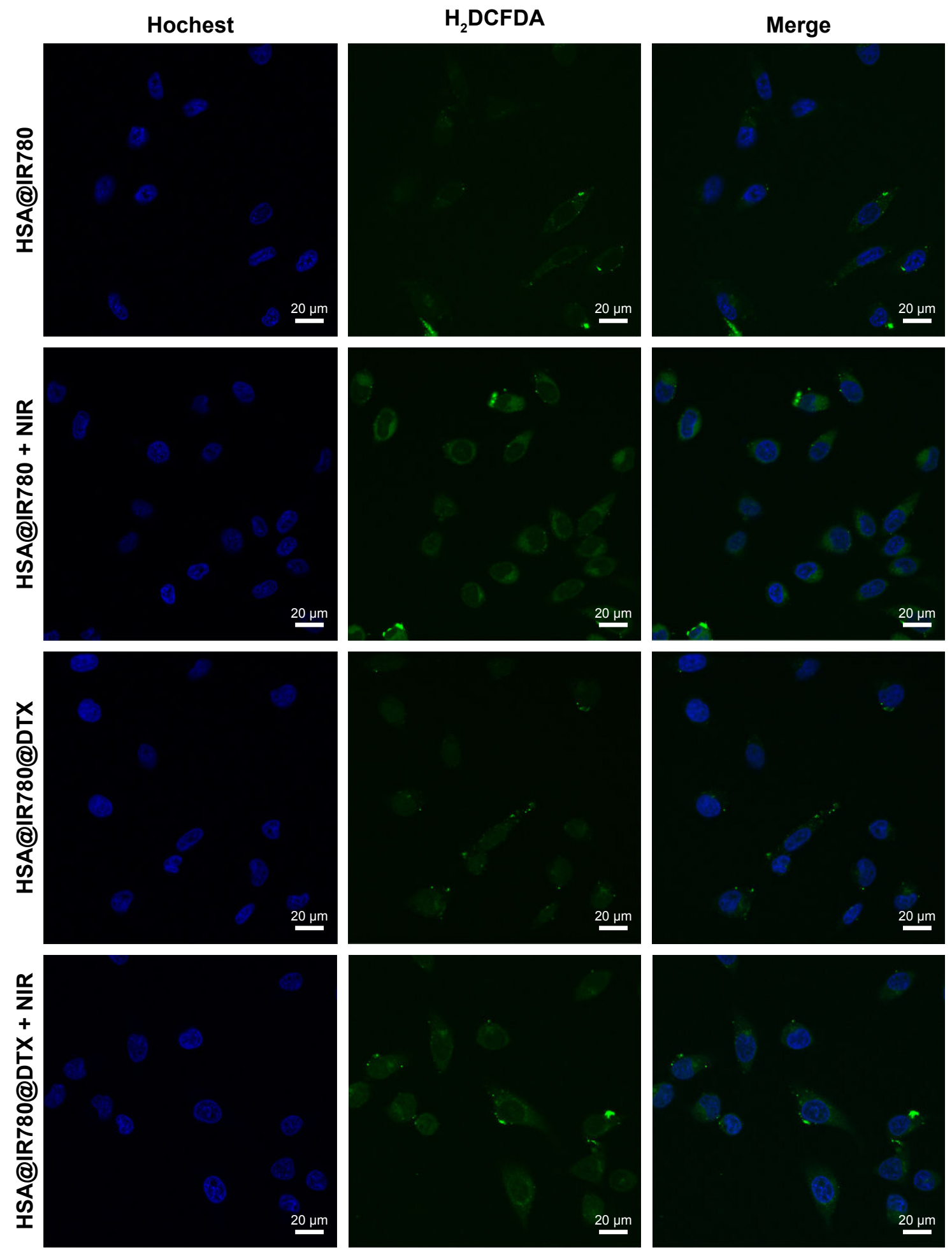

Figure 2 Confocal fluorescence images of ROS generation in MCF-7 cells. From top to bottom: HSA@IR780 NPs, HSA@IR780 NPs plus NIR laser (808 nm, I W/cm², 2 min), HSA@IR780@DTXNPs and HSA@IR780@DTX NPs plus NIR laser. The final concentration of HSA@IR780 NPs (calculated as IR780) was $5 \mu$ g/mL. The green fluorescence color represents oxidatively stressed cells affected with ROS. Fluorescence images were collected at 5I0 560 nm, under an excitation at $504 \mathrm{~nm}$. The scale bar is $20 \mu \mathrm{m}$.

Abbreviations: DTX, docetaxel; HSA, human serum albumin; NIR, near-infrared; NPs, nanoparticles.

Cell uptake of HSA@IR780 NPs and HSA@IR780@DTXNPs

The cellular uptake of HSA@IR780 and HSA@IR780@ DTX NPs in 22RV1 cells were confirmed by a confocal fluorescence microscopy. After $2 \mathrm{~h}$ incubation, strong red fluorescence was observed in the cytoplasm of the cells treated with HSA@IR780 and HSA@IR780@DTX NPs, suggesting that HSA@IR780 and HSA@IR780@DTXNPs 
A

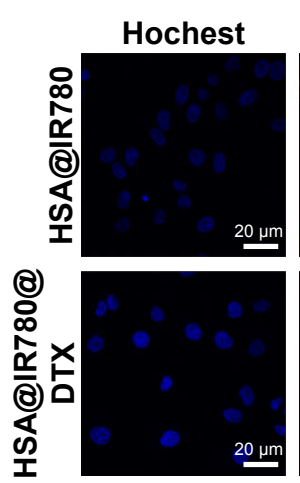

IR780
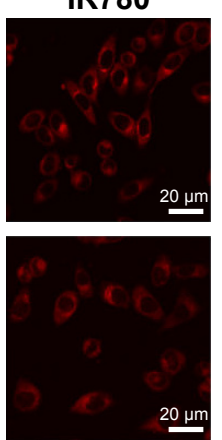
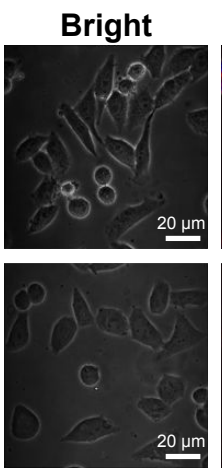

B



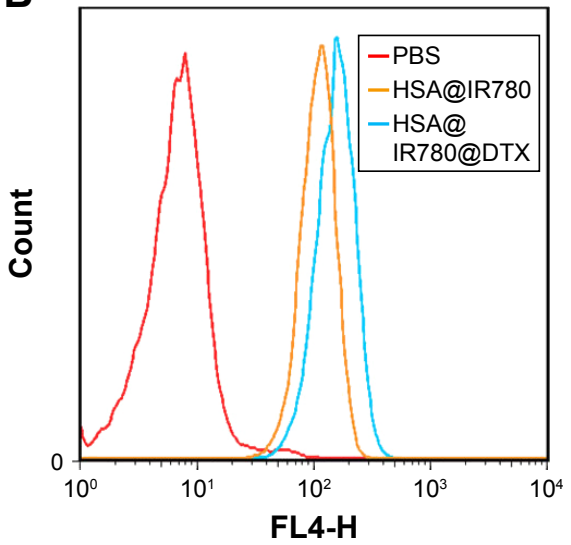

Figure 3 Cellular uptake of HSA@IR780 NPs and HSA@IR780@DTX NPs. (A) Confocal fluorescence images of 22RVI cells localization of HSA@IR780 NPs and HSA@ IR780@DTX NPs 45 min post-incubation. Blue fluorescence indicates positive staining of Hochest and red fluorescence is the fluorescence of IR780. (B) Flow cytometry analysis of 22RVI cell line incubated with PBS, HSA@IR780 NPs, and HSA@IR780@DTX NPs, respectively.

Abbreviations: DTX, docetaxel; HSA, human serum albumin; NPs, nanoparticles; PBS, phosphate buffer saline.

are taken up by the malignant cells (Figure 3A). Flow cytometric analysis (Figure 3B) confirmed that HSA@IR780 NPs and HSA@IR780@DTX NPs are taken up by cells in equal quantities. Our results indicate that DTX did not influence the uptake of HSA@IR780@DTX NPs.

\section{In vitro antitumor therapeutic efficacy of the NPs}

Next, we tested the in vitro antitumor efficacy of HSA@ DTX, HSA@IR780 NPs, and HSA@IR780@DTX NPs in a cancer cell model. Briefly, 22RV1 human prostate cancer cells, cultured under recommended conditions, were firstly incubated with HSA@DTX,HSA@IR780 NPs, and HSA@IR780@DTX NPs for 3 h, then washed with PBS to remove free NPs. Cells were irradiated with $808 \mathrm{~nm}$ laser at a power density of $1 \mathrm{~W} \mathrm{~cm}{ }^{2}$ for $2.5 \mathrm{~min}$. After $24 \mathrm{~h}$, the cell viabilities were determined by the $\mathrm{CCK}-8$ assay to determine the antitumor therapeutic efficacy of the NPs. As shown in Figure 4, the cell viability of cells treated with HSA@ IR780@DTX plus laser irradiation was much lower than that of cells treated with HSA@IR780@DTX without laser irradiation,HSA@DTX with or without laser irradiation, or single photothermal heating induced by HSA@IR780 NPs under NIR laser, suggesting a significant combination effect in comparison to monotherapy. Moreover, HSA@IR780 NPs seem to be affecting cell viability at 1 and $1.5 \mu \mathrm{g} / \mathrm{mL}$ concentrations, probably because IR780 have dark toxicity due to its mitochondria-targeted and hydrophobic property. ${ }^{13,19}$

\section{In vivo imaging of HSA@IR780@ DTX NPs}

To evaluate the feasibility of HSA@IR780@DTX NPs as an ideal nano-photosensitizer, in vivo NIR fluorescence imaging was performed on 22RV1 tumor-bearing mice. NIR fluorescence imaging was applied on tumor-bearing mice which were injected with HSA@IR780@DTX NPs via the tail vein. As shown in Figure 5A, HSA@IR780@ DTX NPs tended to accumulate in the tumor over time, with prominent accumulation in tumor $48 \mathrm{~h}$ post-injection. To further confirm the accumulation of the NPs in tumor, fluorescence in tumor and organs was detected $48 \mathrm{~h}$ after injection of the NPs. The results revealed that the tumor had stronger fluorescent signals, confirming the accumulation of the NPs (Figure 5B). The preferential accumulation in tumors might be due to the enhanced permeability and retention effects.



Figure 4 In vitro cell experiments. Relative viabilities of 22RVI cells after incubation with different concentrations of IR780 and DTX in HSA@DTX, HSA@IR780 NPs, and HSA@IR780@DTXNPs with or without 808 nm laser irradiation (I W/ $/ \mathrm{cm}^{2}, 2 \mathrm{~min}$ ). Abbreviations: DTX, docetaxel; HSA, human serum albumin; NPs, nanoparticles; NIR, near-infrared. 
A

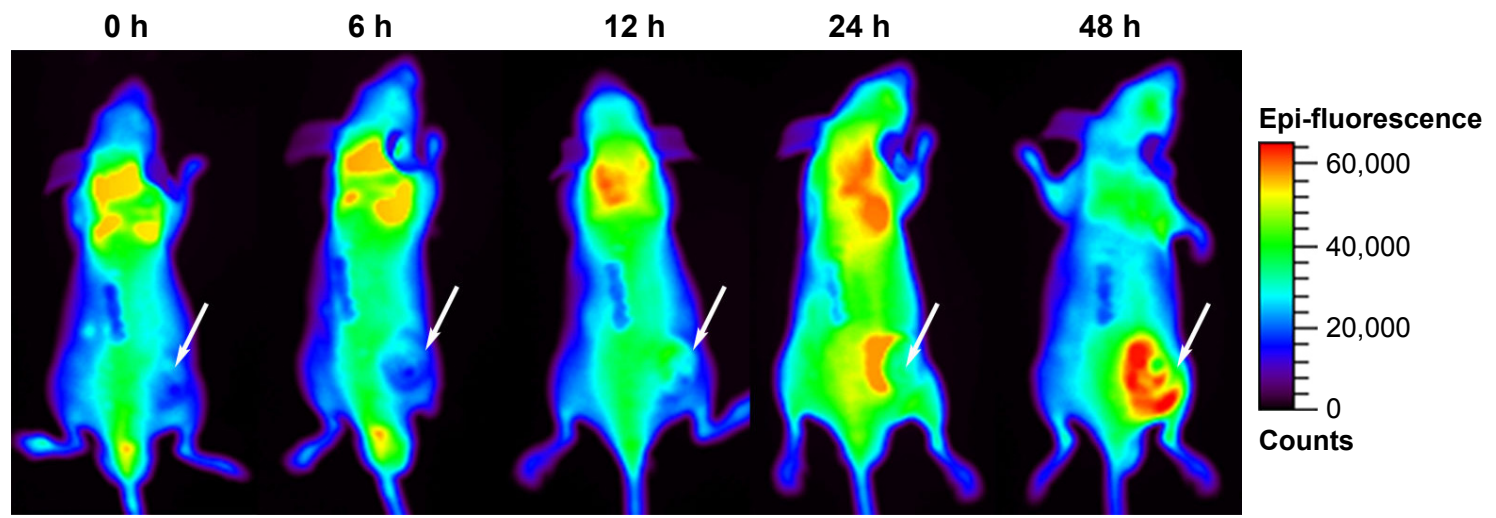

B

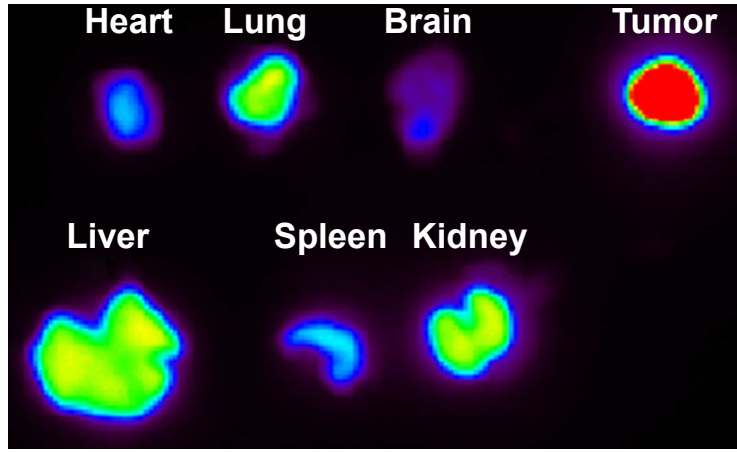

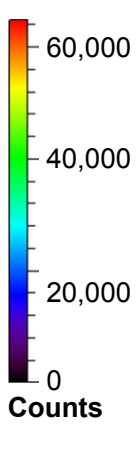

Figure 5 NIR imaging and biodistribution analysis. (A) The fluorescence images of mice with prostate cancer injected with NPs (the white arrows indicated tumor area). (B) Ex vivo fluorescence images of major organs from those mice at $48 \mathrm{~h}$. (C) Semiquantitative biodistribution of NPs in major organs. The data are shown as mean \pm SD ( $\mathrm{n}=3$ ). Abbreviations: NIR, near-infrared; NPs, nanoparticles.

\section{Therapeutic efficacy of NPs in vivo}

Motivated by the high tumor accumulation of HSA@IR780@ DTX, we next investigated the in vivo combined PTT with chemotherapy using the 22RV1 subcutaneous tumor model in mice. Seven groups of 22RV1 tumor-bearing mice with five mice per group were used in this experiment. When the tumor volume reached 100-200 mm³, PBS,HSA@DTX,HSA@ IR780, orHSA@IR780@DTX solutionswere injected into the mice intravenously (doses: IR780 $5 \mathrm{mg} / \mathrm{kg}$, DTX $20 \mathrm{mg} / \mathrm{kg}$ ). The tumors were then irradiated by the $808 \mathrm{~nm}$ laser at a power density of $1 \mathrm{~W} / \mathrm{cm}^{2}$ for $4 \mathrm{~min} 24 \mathrm{~h}$ postinjection when the tumor accumulation of HSA@IR780@ DTX reached its peaked level. An IR thermal camera was used to monitor the temperature change of tumor during laser irradiation. For mice intravenously injected with HSA@ IR780 or HSA@IR780@DTX, the tumor temperatures rapidly increased to $\sim 53^{\circ} \mathrm{C}$ under the laser exposure, whereas mice with PBS injection did not show any significant change under same laser exposure (Figure 6A). However, even though PTT alone induced tumor damage, it could not completely destruct subcutaneous 4T1 tumors based on previous reports. ${ }^{20}$ The tumor sizes were then measured by a caliper. At 2 days post-injection,HSA@IR780@DTX plus laser and HSA@IR780 plus laser-treated mice demonstrated a severe tumor necrosis at the site of the laser irradiation. A similar necrosis was not observed in the other groups (Figure 7A). After 9 days, it was apparent that the necrotic scar tissue was healed and that normal tissue had begun to regenerate in the HSA@IR780@DTX plus laser-treated mice. The therapeutic efficacy of each treatment was monitored by evaluating the tumor volumes for 18 days (Figure 6B). Remarkably, tumors on mice treated with HSA@IR780@DTX plus NIR laser irradiation were completely inhibited without a single case of regrowth in the period up to 18 days. In contrast, tumors on mice with chemotherapy alone (HSA@DTX, HSA@ IR780@DTX without laser) or with PTT alone (HSA@IR780 with laser) showed a moderate growth inhibition effect. Particularly, tumor size in the HSA@IR780 with laser group was similar to that in HSA@IR780@DTX with laser group in the first 3 days after the treatment. However, 3 days later, the HSA@IR780 with laser group showed tumor regrowth gradually and HSA@IR780@DTX with laser group showed continuous tumor growth inhibition effect, owing to the combination effect of PTT and chemotherapy. H\&E staining of tumor slices collected 18 days post-treatment revealed that tumor cells were severely destroyed after combination therapy (HSA@IR780@DTX plus NIR laser),while those after single therapy were only partially damaged (Figure 7B). 

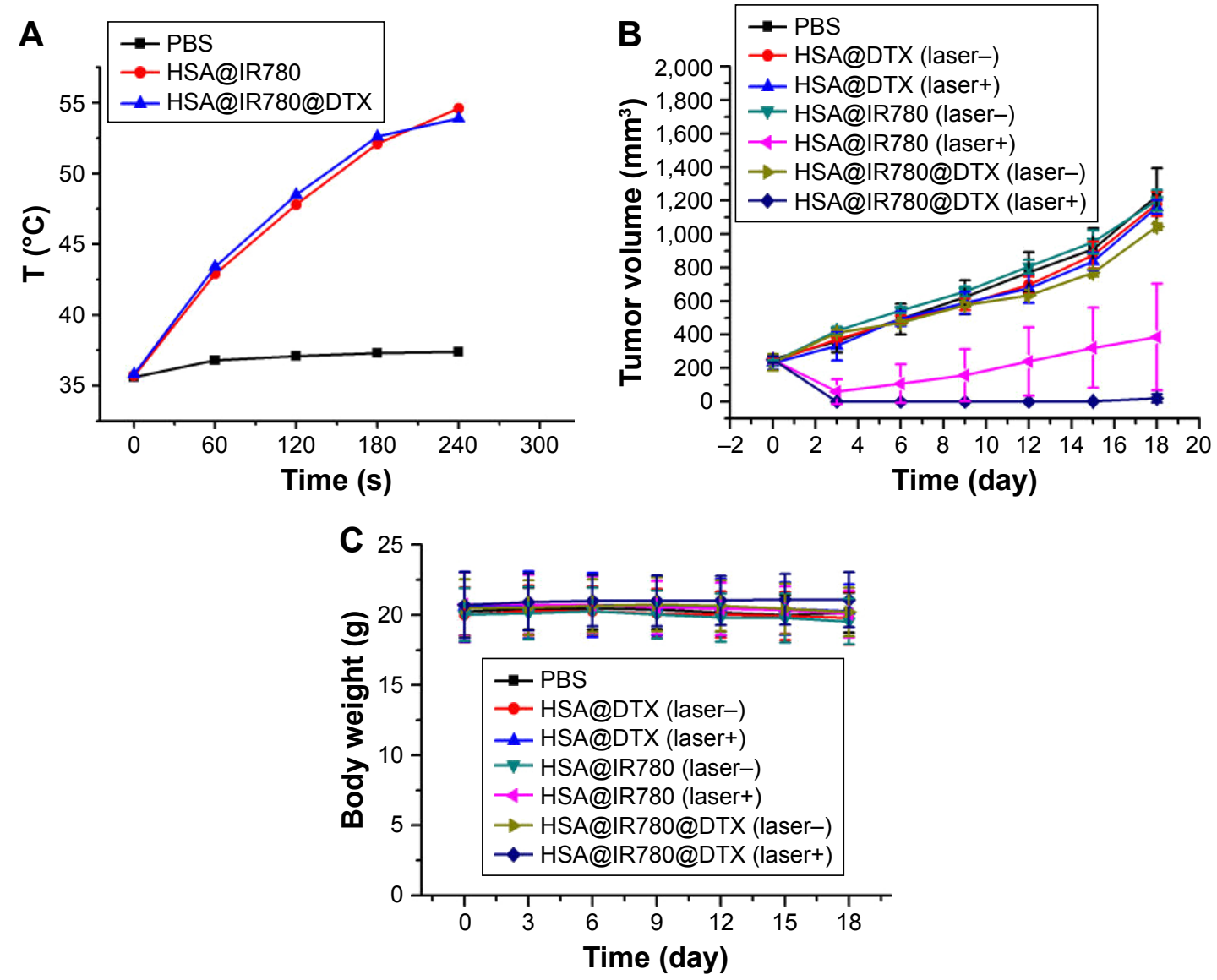

Figure 6 Imaging guided combination therapy on a subcutaneous tumor model. (A) Heating curves of tumor-bearing mice with PBS, HSA@IR780 NPs and HSA@IR780@ DTX NPs. Data are expressed as mean \pm SD $(n=3)$. (B) The tumor growth curves of different group of mice after various treatments indicated (five mice per group). Error bars were based on standard errors of the mean. (C) Body weight of mice in different group after treatment.

Abbreviations: DTX, docetaxel; HSA, human serum albumin; NPs, nanoparticles; PBS, phosphate buffer saline.

A
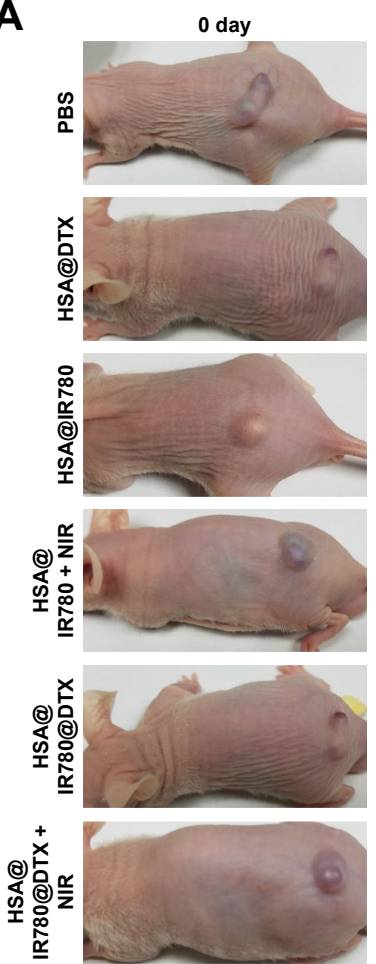

2 days
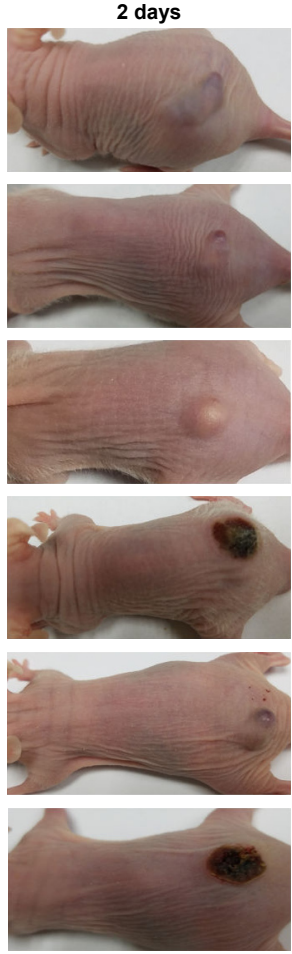

9 days
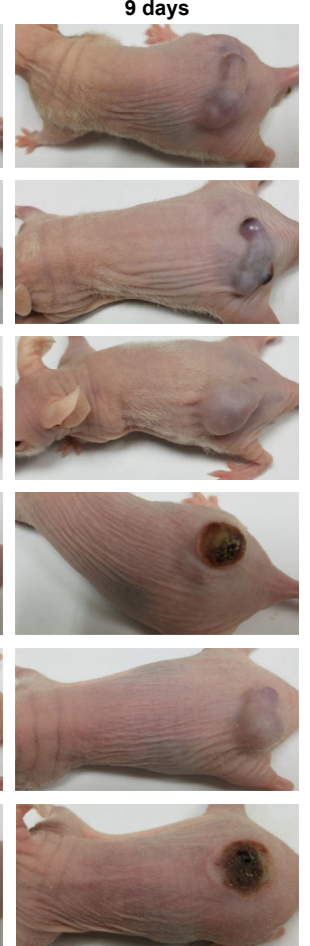
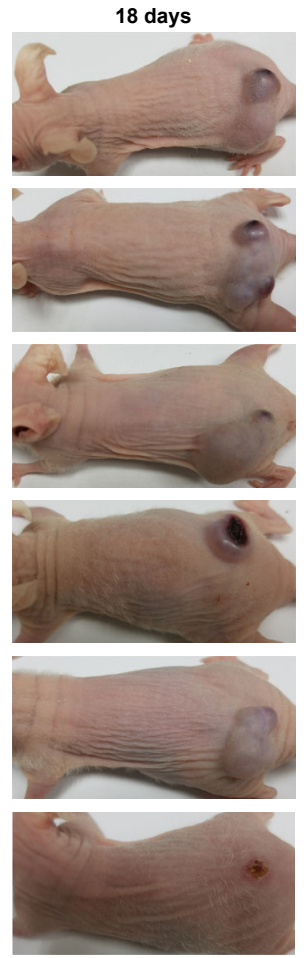

Figure 7 (Continued) 
B
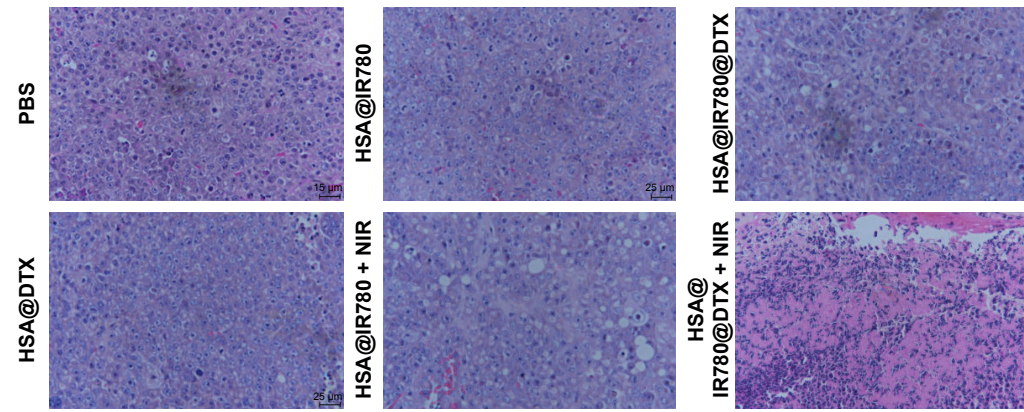

C


Kidney

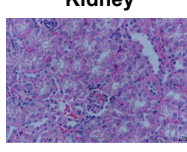

Spleen
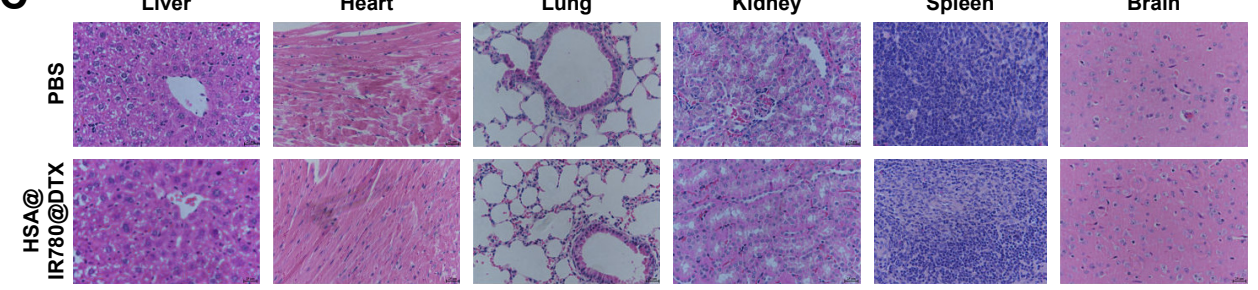

Figure 7 Therapeutic efficacy and biosafety evaluation of formed nanoparticles in vivo.

Notes: (A) Representative images of mice bearing prostate cancer pre-treatment and after treatments, (B) the corresponding H\&E-stained tumor sections, (C) H\&E staining of major organs after the treatment of nanoparticles.

Abbreviations: DTX, docetaxel; HSA, human serum albumin; NIR, near-infrared; PBS, phosphate buffer saline.

Neither body weight loss nor animal death was observed in each experimental group (Figure 6C). H\&E stained images of the primary organs from HSA@IR780@DTX-injected mice 18 days post-injection indicated no appreciable abnormality or noticeable organ damage (Figure 7C). Therefore, our results demonstrate an excellent synergistic therapeutic effect of the combined PTT and chemotherapy delivered by HSA@IR780@DTX, which did not show significant toxic side effect on the treated animals.

\section{Conclusion}

We developed a simple method to encapsulate hydrophobic IR780 and DTX into HSA to form multifunctional HSA@IR780@DTX NPs for the combination therapy of PTT and PDT with chemotherapy in the treatment of castration-resistant prostate cancer. These new HSA@ IR780@DTX NPs exhibit great advantages in both imaging and combined therapy, suggesting a great potential in clinical translation. Based on such biocompatible theranostic agent, it is hoped that imaging-guided combined PTT and/or PDT therapy with chemotherapy would bring new perspectives to the future fight against castration-resistant prostate cancer.

\section{Acknowledgments}

This work was supported by grants from the National Natural Science Foundation of China (Nos 81572519, 81502203,
81602221) and Jiangsu Provincial Medical Youth Talent (QNRC2016017).

\section{Disclosure}

The authors report no conflicts of interest in this work.

\section{References}

1. Gundem G, Van Loo P, Kremeyer B, et al. The evolutionary history of lethal metastatic prostate cancer. Nature. 2015;520(7547):353-357.

2. Heidenreich A, Bastian PJ, Bellmunt J, et al. EAU guidelines on prostate cancer. Part II: Treatment of advanced, relapsing, and castration-resistant prostate cancer. Eur Urol. 2014;65(2):467-479.

3. Petrylak DP, Vogelzang NJ, Budnik N, et al. Docetaxel and prednisone with or without lenalidomide in chemotherapy-naive patients with metastatic castration-resistant prostate cancer (MAINSAIL): a randomised, double-blind, placebo-controlled phase 3 trial. Lancet Oncol. 2015;16(4):417-425.

4. Antonarakis ES, Lu C, Luber B, et al. Androgen receptor splice variant 7 and efficacy of taxane chemotherapy in patients with metastatic castration-resistant prostate cancer. JAMA Oncol. 2015;1(5): 582-591.

5. Ganju A, Yallapu MM, Khan S, Behrman SW, Chauhan SC, Jaggi M. Nanoways to overcome docetaxel resistance in prostate cancer. Drug Resist Updat. 2014;17(1-2):13-23.

6. Koukourakis MI, Giatromanolaki A, Panteliadou M, et al. Lactate dehydrogenase 5 isoenzyme overexpression defines resistance of prostate cancer to radiotherapy. Br J Cancer. 2014;110(9):2217-2223.

7. Tannock IF, Fizazi K, Ivanov S, et al; VENICE Investigators. Aflibercept versus placebo in combination with docetaxel and prednisone for treatment of men with metastatic castration-resistant prostate cancer (VENICE): a phase 3, double-blind randomised trial. Lancet Oncol. 2013;14(8):760-768.

8. Karantanos T, Corn PG, Thompson TC. Prostate cancer progression after androgen deprivation therapy: mechanisms of castrate resistance and novel therapeutic approaches. Oncogene. 2013;32(49):5501-5511. 
9. D’Amico AV, Chen MH, Renshaw A, Loffredo M, Kantoff PW. Long-term follow-up of a randomized trial of radiation with or without androgen deprivation therapy for localized prostate cancer. JAMA. 2015;314(12):1291-1293.

10. Meng Z, Wei F, Wang R, et al. NIR-laser-switched in vivo smart nanocapsules for synergic photothermal and chemotherapy of tumors. Adv Mater. 2016;28(2):245-253.

11. Chen W, Ouyang J, Liu H, et al. Black phosphorus nanosheet-based drug delivery system for synergistic photodynamic/photothermal/ chemotherapy of cancer. Adv Mater. 2017;29(5):1603864.

12. Espinosa A, Silva AK, Sánchez-Iglesias A, et al. Cancer cell internalization of gold nanostars impacts their photothermal efficiency in vitro and in vivo: toward a plasmonic thermal fingerprint in tumoral environment. Adv Healthc Mater. 2016;5(9):1040-1048.

13. Jiang C, Cheng H, Yuan A, Tang X, Wu J, Hu Y. Hydrophobic IR780 encapsulated in biodegradable human serum albumin nanoparticles for photothermal and photodynamic therapy. Acta Biomater. 2015;14:61-69.

14. Ding D, Tang X, Cao X, Wu J, Yuan A, Qiao Q, et al. Novel selfassembly endows human serum albumin nanoparticles with an enhanced antitumor efficacy. AAPS PharmSciTech. 2014;15(1):213-222.
15. Kratz F. A clinical update of using albumin as a drug vehicle - a commentary. J Control Release. 2014;190:331-336.

16. Chen Q, Liu Z. Albumin carriers for cancer theranostics: a conventional platform with new promise. Adv Mater. 2016;28(47):10557-10566.

17. Yan F, Duan W, Li Y, et al. NIR-laser-controlled drug release from DOX/IR-780-loaded temperature-sensitive-liposomes for chemophotothermal synergistic tumor therapy. Theranostics. 2016;6(13): 2337-2351.

18. Yue C, Liu P, Zheng M, et al. IR-780 dye loaded tumor targeting theranostic nanoparticles for NIR imaging and photothermal therapy. Biomaterials. 2013;34(28):6853-6861.

19. Zhang E, Luo S, Tan X, Shi C. Mechanistic study of IR-780 dye as a potential tumor targeting and drug delivery agent. Biomaterials. 2014; 35(2):771-778.

20. Chen Q, Liang C, Wang C, Liu Z. An imagable and photothermal "Abraxane-like" nanodrug for combination cancer therapy to treat subcutaneous and metastatic breast tumors. Adv Mater. 2015;27(5):903-910.
International Journal of Nanomedicine

\section{Publish your work in this journal}

The International Journal of Nanomedicine is an international, peerreviewed journal focusing on the application of nanotechnology in diagnostics, therapeutics, and drug delivery systems throughout the biomedical field. This journal is indexed on PubMed Central, MedLine, CAS, SciSearch $®$, Current Contents $\AA /$ Clinical Medicine,

\section{Dovepress}

Journal Citation Reports/Science Edition, EMBase, Scopus and the Elsevier Bibliographic databases. The manuscript management system is completely online and includes a very quick and fair peer-review system, which is all easy to use. Visit http://www.dovepress.com/ testimonials.php to read real quotes from published authors. 